\title{
EL EFECTO VOLTA. UN CASO DE ESTUDIO SOBRE LA PRODUCCIÓN DE EFECTOS SENSIBLES Y LOS PROCESOS DE TEORIZACIÓN EN CIENCIAS
}

\author{
MARINA GARZÓN BARRIOS ${ }^{*}$ \\ https://orcid.org/0000-0002-1468-3629 \\ LILIANA TARAZONA VARGAS ${ }^{* *}$ \\ https://orcid.org/0000-0003-3752-3879 \\ SANDRA SANDOVAL OSORIO ${ }^{1 * *}$ \\ https://orcid.org/0000-0001-6575-5808 \\ JOSÉ FRANCISCO MALAGÓN SÁNCHEZ ${ }^{\cdots \cdots *}$ \\ https://orcid.org/0000-0002-0952-2521 \\ MARÍA MERCEDES AYALA MANRIQUEE ${ }^{1 \cdots *}$ \\ https://orcid.org/0000-0002-3045-4914
}

RESUMEN: En este artículo presentamos algunas reflexiones sobre las relaciones entre la percepción, la producción de efectos sensibles y los procesos de síntesis teórica en la construcción de conocimiento en ciencias. Para explicitar estas relaciones, realizamos un estudio histórico-crítico del trabajo de Alessandro Volta (1745-1827) sobre la pila voltaica y el circuito eléctrico. Volta detecta y reproduce ciertos efectos eléctricos mediante experimentos que le permiten percibir la continuidad y direccionalidad del fluido eléctrico mientras constituye proposiciones teóricas sobre la electricidad. Este caso ilustra el proceso constructivo de conocimiento que es necesario considerar al establecer criterios para la enseñanza de las ciencias.

Palabras clave: Enseñanza de las ciencias. Procesos de teorización en ciencias. Efecto Volta.

\section{EFEITO VOLTA. UM ESTUDO DE CASO SOBRE A PRODUCC̃̃O DE EFEITOS SENSÍVEIS E OS PROCESSOS DE TEORIZAÇÃO NA CIÊNCIAS}

RESUM0: Neste artigo, apresentamos algumas reflexões sobre as relações entre a percepção, produção de efeitos sensíveis e os processos de síntese teórica na construção do conhecimento em ciências. Para explicar estas relações, realizamos um estudo histórico-crítico da obra de
*Estudios doctorales en Didáctica de las Ciencias Experimentales y Matemáticas. Universidad de Barcelona. Magister en Historia de las Ciencias. Grupo de investigación Estudios Histórico-Críticos y Enseñanza de las Ciencias $\mathrm{EHC}^{\wedge} \mathrm{EC}$. Docente investigadora Departamento de Física. Universidad Pedagógica Nacional. Bogotá D.C, Colombia.

E-mail: mgarzonb@pedagogica.edu.co

* * Doctora en Ciencias: Especialidad Investigaciones Educativas. Centro de Estudios Avanzados IPN México. Maestría en Metodología de la Ciencia. Grupo de investigación Estudios Histórico-Críticos y Enseñanza de las Ciencias EHC^ $E C$. Docente investigadora Departamento de Física. Universidad Pedagógica Nacional. Bogotá D.C, Colombia.

E-mail: Itarazonav@pedagogica.edu.co

*** Magister en Educación. Estudios doctorales en Educación Grupo de investigación Estudios Histórico-Críticos y Enseñanza de las Ciencias EHC^EC. Docente investigadora Departamento de Química Universidad Pedagógica Nacional. Bogotá D.C, Colombia.

E-mail: ssandoval@pedagogica.edu.co

**** Magister en Docencia de la Física. Grupo de investigación Estudios Histórico-Críticos y Enseñanza de las Ciencias EHC^ $\mathrm{EC}$. Docente Investigador

' Universidad Pedagógica Nacional, Departamento de Física, Bogotá DC. - Colombia 
Alessandro Volta (1745-1827) sobre a pilha voltaica e o circuito elétrico. Volta detecta e reproduz certos efeitos elétricos através de experimentos que lhe permitem perceber a continuidade e a direcionalidade do fluido elétrico ao mesmo tempo em que constituem proposições teóricas sobre a eletricidade. Este caso ilustra o processo construtivo de conhecimento que é necessário considerar ao estabelecer critérios para o ensino de ciências.

Palavras-chaves: Ensino de ciências. Processos de teorização em ciências. Efeito Volta.

\section{THE VOLTA EFFECT. A CASE STUDY ON THE PRODUCTION OF SENSITIVE EFFECTS AND THEORIZING PROCESSES IN SCIENCE.}

ABSTRACT: In this paper, we present some reflections on the relations between perception, the production of sensitive effects and the processes of theoretical synthesis in the construction of knowledge in science. To illustrate these relations, we developed a historical-critical study of the work of Alessandro Volta (1745-1827) on the voltaic battery and the electrical circuit. Volta detects and reproduces certain electrical effects through experiments that allow him to perceive the continuity and directionality of the electric fluid while constituting theoretical propositions about electricity. This case exemplifies the constructive process of knowledge that is necessary to consider when establishing criteria for science education. Keywords: Science Education. Theorization processes in science. Volta Effect.
Departamento de Física. Universidad Pedagógica Nacional. Bogotá D.C, Colombia. E-mail: jmalagon@pedagogica.edu.co

***** Master of Science Physics Indiana University. Docente investigadora jubilada. Universidad Pedagógica Nacional. Departamento de Física. Bogotá D.C, Colombia. E-mail: ayalam49@gmail.com 


\section{INTRODUCCIÓN}

Los procesos de conceptualización, de estructuración de proposiciones, de descripciones o de explicaciones que emergen cuando se estudia un fenómeno natural, han sido identificados por nosotros como un campo de estudio necesario para la comprensión del proceso constructivo de la actividad científica y, por lo tanto, necesario para la enseñanza de las ciencias cuando se tiene como objetivo generar escenarios para la construcción de conocimiento científico en el aula.

En este campo hemos identificado una compleja y estrecha relación entre: - la forma de percibir un fenómeno,

- la necesidad de hacer explícita esta percepción por medio de proposiciones y argumentaciones, que en muchos casos conducen a la producción de efectos que permiten ampliar y reproducir la percepción,

- y la síntesis que se concreta en la concatenación y estructuración de conceptos, de instrumentos y de enunciados teóricos.

Por lo cual, hemos abordado una perspectiva fenomenológica (AYALA MANRIQUE y otros, 2013, 2014; 2018a; 2018b) que establece un vínculo indisoluble entre la percepción, la producción lingüística y la organización conceptual. Consideramos que es importante examinar cómo se constituyen formas lógicas y organizaciones teóricas a partir de este vínculo, pues permitirá proponer criterios que guíen los procesos de enseñanza.

Hemos identificado que en la enseñanza de las ciencias existe una fuerte tendencia a tomar como objetos de estudio los productos o resultados de la ciencia que se presentan en forma de leyes, teorías e incluso de métodos, que reemplazan el estudio sobre los fenómenos; esta tendencia se encuentra en constante tensión con propuestas centradas en los procesos científicos (ERDURAN; DAGHER, 2014, p. 67). Consideramos que, si se inicia el proceso de enseñanza partiendo desde las leyes, de los modelos o de las teorías, se restringe el proceso constructivo en el aprendizaje de las ciencias, que es, ante todo, un proceso cognitivo, intersubjetivo y cultural.

Por ejemplo, cuando se ingresa en el estudio de los circuitos eléctricos - que es el problema que abordaremos en relación con el efecto voltaico - habitualmente el punto de partida es la ley de $\mathrm{Ohm}$. Con esta ley aparecen términos como voltaje, corriente, resistencia eléctrica, y también aparecen definiciones: la corriente eléctrica es definida en términos de la circulación de electrones en los diferentes elementos del circuito. En este caso, la imagen del electrón como partícula con carga negativa, que se desplaza en los materiales conductores, es el eje de la explicación. ${ }^{1}$

Pensamos que cuando esta imagen electrónica se enuncia para ser el lugar de partida para la enseñanza, los razonamientos de los estudiantes son estructurados a partir de una serie de definiciones que no emergen de aquello que puedan observar y describir sobre los fenómenos de estudio, y las afirmaciones se convierten en definiciones a priori representadas mediante entidades que en ese estadio de conocimiento parecen metafísicas. ${ }^{2}$

En estos casos, el aprendizaje se encuentra en el dominio del resultado científico, de la ley o del modelo que representa el fenómeno en estudio, y no se encuentra en el dominio de su constitución. Como consecuencia, hay menos posibilidades para reflexionar sobre la actividad científica, para identificar los 
problemas de estudio, para seguir los razonamientos lógicos y criterios de análisis que llevaron a esta forma particular de organización teórica; de este modo, la enseñanza se presenta y desarrolla de forma deductiva o axiomática. Por su parte, este punto de partida tiene la ventaja de que la ley o el modelo han sintetizado los diferentes casos particulares y se adquiere una certeza dentro de la teoría científica, en la cual la experimentación cumple un papel demostrativo y permite ejemplificar.

Sin embargo, vemos como una gran desventaja que al ser proposiciones teóricas presentadas sin el contexto que les ha dado origen, se convierten en problemas de estudio descontextualizados, y ajenos también al campo de experiencias de los estudiantes, de modo que ellos podrán tener dificultades conceptuales para seguir los razonamientos constitutivos de esas proposiciones teóricas, limitando el alcance de su comprensión.

Desde luego, esto no significa que planteemos que las teorías científicas no tengan presencia en la enseñanza, por el contrario, siendo conscientes del valor de la estructuración de leyes y teorías en la constitución de la actividad científica, consideramos necesario identificar cómo se generan y concatenan enunciados, cómo estos enunciados se constituyen en formas lógicas y en organizaciones que dan cuenta de un conjunto de fenómenos, que se generalizan y sintetizan inicialmente en forma de leyes experimentales, y posteriormente, en estructuras teóricas consolidadas (DUHEM, 2003).

Nosotros consideramos que un elemento imprescindible en el aprendizaje de las ciencias es el proceso que permite emitir enunciados o proposiciones lógicas sobre el mundo perceptible porque este proceso indica las comprensiones adquiridas al actuar sobre ese mundo; por este motivo, ilustraremos dicho proceso mediante el caso de la organización del efecto voltaico.

Así pues, en este artículo se hace un análisis sobre el trabajo de Alessandro Volta (1745-1827) alrededor de la pila voltaica y sobre el circuito eléctrico, para señalar algunas reflexiones sobre los procesos de síntesis teórica que tiene origen en la percepción ${ }^{3}$ y la producción de efectos sensibles, reflexiones dirigidas hacia el desarrollo de criterios epistemológicos para la enseñanza de las ciencias.

Mediante este estudio se muestra la estrecha relación de estructuración entre percepción - lenguaje, experimentación - formalización y elaboración de principios teóricos, aspectos que nos permiten argumentar nuestros hallazgos respecto a los procesos de teorización en ciencias.

\section{PRODUCCION DE EFECTOS SENSIBLES: EL EFECTO VOLTA}

Los estudios histórico-críticos de las ciencias han permitido reconocer momentos importantes del trabajo de Volta, que se discutirán en este apartado. En primer lugar, la construcción de la pila y los efectos que dan origen a la idea de circuito, y en segundo lugar, la definición de electricidad a partir de la unificación de diversos fenómenos eléctricos. Mediante estos dos momentos presentaremos la constitución del efecto Volta, que será el eje de nuestras reflexiones sobre los procesos de teorización. 


\subsection{La pila y los efectos que dan origen a la idea de circuito eléctrico}

Hacia finales del siglo XVIII, Luigi Galvani (1737-1798) había presentado sus experimentos sobre la "electricidad animal". En su obra: La fuerza eléctrica en el movimiento muscular [De viribus electricitatis in motu musculari (1791)], mostraba que al tocar con un bisturí el nervio de la pata de una rana que colgaba de un metal, y mediante la acción de una chispa eléctrica entre los dos metales, la electricidad de los animales se manifestaba en forma de movimientos bruscos y frecuentes (GALVANI, 1937). Así, manteniendo una conexión en circuito entre estos elementos (metales, tejido muscular, nervios, chispas) era posible excitar el movimiento de animales tanto muertos como vivos. Galvani declaraba que la electricidad allí percibida provenía de los tejidos y los músculos y la consideraba propia de los animales.

Figura 1. Aparato de Columna. Tomado de VOLTA (1800b)

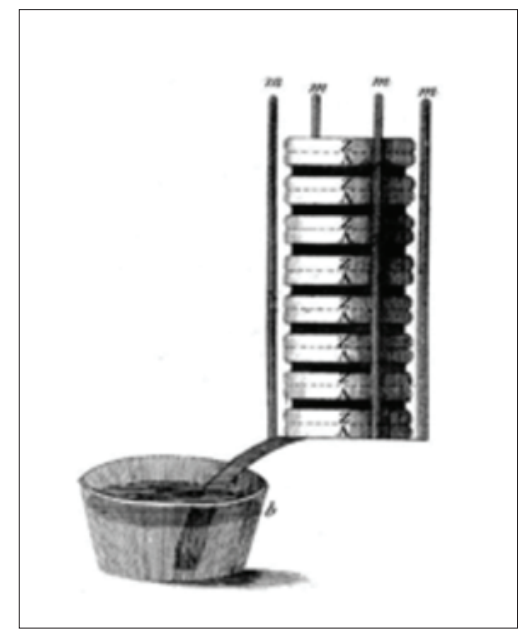

Por su parte, Alessandro Volta (1745-1827), atraído por los trabajos de Galvani, había concluido que la electricidad exhibida en las contracciones de las patas de las ranas, y la presente en otros aparatos eléctricos, se producía en relación con el círculo formado por los metales puestos en contacto, y, por lo tanto, no era una electricidad propia o particular de los animales. ${ }^{4}$

El origen de la conclusión de Volta tuvo lugar con el diseño de un aparato electromotor (Figura 1.) que hoy día conocemos como pila voltaica. La pila está constituida por la disposición de las parejas de placas metálicas distintas entre las cuales se encuentran cartones humedecidos, y al tocar simultáneamente sus extremos metálicos, se producen algunos efectos.

En esta etapa de su trabajo, Volta describe las sensaciones que se producen en los órganos del cuerpo humano, que él utilizó como su principal sensor. Veamos un ejemplo de estas descripciones: ${ }^{5}$

Los efectos sensibles a nuestros órganos que produce un aparato formado por 40 o 50 pares de placas, (e incluso menor si uno de los metales es plata o cobre, el otro zinc), no 
se reducen simplemente a conmociones: la corriente del fluido eléctrico, movido y actuado, por un tal número y especies de conductores distintos, plata, zinc y agua, dispuestos alternativamente de la manera descrita, no provoca sólo contracciones y espasmos en los músculos, convulsiones más o menos violentas en los miembros que atraviesa en su recorrido, sino que irrita también los órganos del gusto, de la vista, del oído y del tacto, propiamente dicho, produciendo sensaciones propias de cada uno. (VOLTA, 1800a, p. 776)

Es posible apreciar que la expresión corriente del fluido eléctrico aparece como causa de los efectos: convulsión, contracción, espasmo, irritación, etc, cuando se establece contacto entre la primera y la última pareja del apilamiento. Además, ese fluido "atraviesa en su recorrido" los miembros, y es “movido y actuado" debido a la distribución de platos metálicos: con una mayor cantidad de parejas de placas (plata o cobre y zinc) se aumenta el efecto y se facilita la percepción de la sensación.

Observemos ahora el siguiente fragmento:

Aplico [en la piel ], como decía, con un poco de presión, alguna de estas partes delicadas [frente, punta de la nariz, párpados], bien humedecidas, contra la punta de un hilo metálico, que comunicará convenientemente con el otro extremo de dicho aparato, siento, en el momento en que se cierra el círculo conductor, en el lugar de la piel en el que se produce el contacto, y un poco más allá, un golpe y un pinchazo, que pasa rápidamente, y se repite tantas veces como se interrumpe y se restablece el círculo... Pero, si todas las comunicaciones continúan sin estas alternativas, sin la menor interrupción del círculo, por algunos instantes no siento nada; pasados los cuales, empieza a sentirse en la parte aplicada al final del hilo metálico, otra sensación, un dolor agudo, (sin sacudidas), limitado precisamente a los puntos de contacto, una abrasión, no solamente continua, sino que va constantemente en aumento, hasta el punto de convertirse en un corto espacio de tiempo en insoportable, y que no cesa si no se interrumpe el círculo. (VOLTA, 1800a, p. 776)

Volta hace un bosquejo de la idea de circuito cerrado, o en sus palabras: círculo conductor. Establece las condiciones de producción y detección de los diversos efectos sensibles, a partir de expresiones como: "siento, en el momento en que se cierra el círculo conductor", el efecto "se repite tantas veces como se interrumpe y se restablece el círculo", "no cesa si no se interrumpe el círculo". Es de notar, también, que la duración y la interrupción de la sensación son esenciales para manifestar una comunicación conveniente que garantiza la continuidad de la corriente cuando se establece un circuito.

Volta continúa:

¿Existe prueba más evidente de la continuidad de la corriente eléctrica, durante todo el tiempo que las comunicaciones de los conductores que forman el círculo continúan y de que, solamente interrumpiendo el círculo, una tal corriente cesa de existir? Esta circulación sin fin del fluido eléctrico puede parecer una paradoja, puede no ser explicable; pero no es menos verdadera y real [...]. (VOLTA, 1800a, p. 776) 
Esta organización sobre el circuito implica identificar las condiciones de producción y la duración de los efectos sensibles que él mismo experimenta, se produce evidencia sobre "la continuidad de la corriente eléctrica", lo que lleva a pensar en "circulación sin fin del fluido eléctrico".

Para nosotros, estas expresiones y afirmaciones representan una organización conceptual que se ha ido estructurando a través de proposiciones que enmarcan una síntesis conceptual o, en otras palabras, una síntesis teórica. Cada término nuevo o cada expresión son derivados de una organización perceptual que es inferencial, que es lógica, que es concluyente y que, además, lleva a proponer nuevas acciones.

Como veremos ahora, Volta se dedicó a examinar otro círculo conductor constituido por una disposición de tazas metálicas unidas a través de arcos metálicos (Figura 2.):

Figura 2. Corona de tazas. Tomado de VOLTA (1800b).

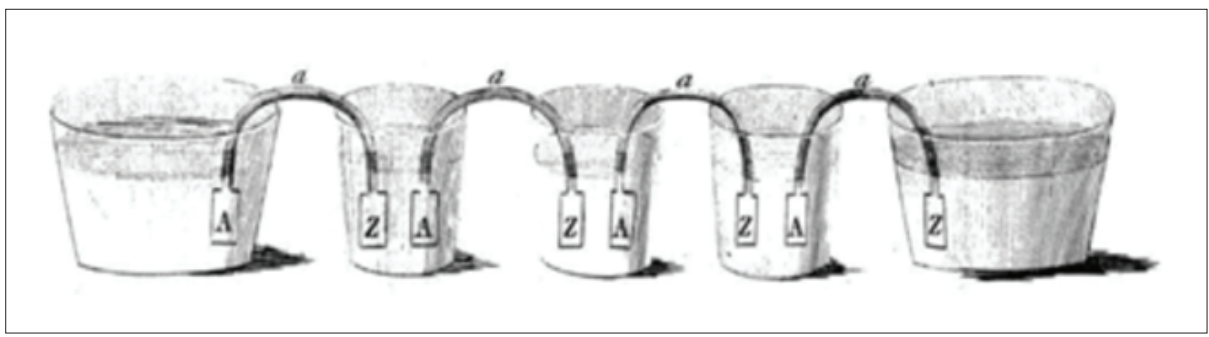

Si la cadena se interrumpe en alguna parte, sea porque falte agua en alguna de las tazas, sea porque uno de los arcos metálicos ha sido quitado, o porque haya sido separado en las dos piezas [constituyentes], no tendréis ninguna conmoción sumergiendo un dedo en el agua del primer, y otro en la del último vaso; la tendréis sin embargo más fuerte o más débil, según las circunstancias, (dejando estos dedos sumergidos), en el momento en que se restablezca la comunicación interrumpida, en el momento en que otra persona sumerja en las dos tazas en la que falta el arco, dos de sus dedos (que sufrirán también una pequeña conmoción), o mejor, sumerja ese mismo arco que había sido quitado, u otro cualquiera; y, en el caso del arco separado en dos piezas, en el momento en que se restablezca el contacto mutuo; (así la conmoción será mayor que de otra manera) [...] (VOLTA, 1800a, p. 772).

En estos fragmentos Volta refuerza sus afirmaciones sobre la necesidad del circuito. Para mantenerlo se requiere de los dedos sumergidos en la primera y última taza que hacen parte de ese círculo, tanto así que podrían remplazar uno de los arcos metálicos que comunican las tazas, también se requiere del agua que contienen las tazas porque hace posible la continuidad de la corriente. La gradación en la intensidad ${ }^{6}$ de la sensación es un aspecto que también se resalta en la descripción de Volta: "la tendréis sin embargo más fuerte o más débil, según las circunstancias", la sensación ya no es solamente larga o corta en duración, es además fuerte o débil.

En el primer caso del aparato de torre, los efectos sensibles: las conmociones, irritaciones, pinchazos, no suceden si no hay una distribución adecuada de los platos, el hilo metálico, la frente, la nariz o los párpados humedecidos. En el 
segundo caso de la corona de tazas, los efectos no se producen si no hay una disposición particular de tazas, arcos metálicos y dedos sumergidos en el agua. En las dos distribuciones se necesita vincular dos metales diferentes: el primer y el último elemento, un círculo que se cierra con los dedos o los órganos y se encuentra "limitado precisamente a los puntos de contacto".

La forma de construir y comunicar el circuito eléctrico que aparece como condición para la producción de efectos no es una elección, menos aún, una elección arbitraria o una escogencia al azar que se puede o no se puede establecer, por el contrario, es una acción intencional y obligatoria para producir fluido eléctrico. Las descripciones realizadas aquí muestran que los efectos se formalizan, en tanto se buscan las condiciones para producirlos y para diferenciarlos en intensidad. Los nuevos términos como "círculo conductor" implican una organización de esta experiencia perceptiva de la cual consideramos que resulta una abstracción teórica que podría ser expresada como un principio: para que fluy a electricidad debe mantenerse un circuito cerrado de metales diferentes en contacto con un conductor búmedo.

\subsection{De las descargas al circuito conductor}

La electricidad artificial que Volta había experimentado con la pila de discos metálicos podía producir conmociones tan intensas como las que se percibían al utilizar las botellas de Leyden (Figura 3). ${ }^{7}$ Estas botellas al estar electrificadas producen descargas tan intensas como breves, generando a su paso el destello de una chispa eléctrica.

Figura 3. Botella de Leyden. Tomado de GANOT (1859, p. 457)

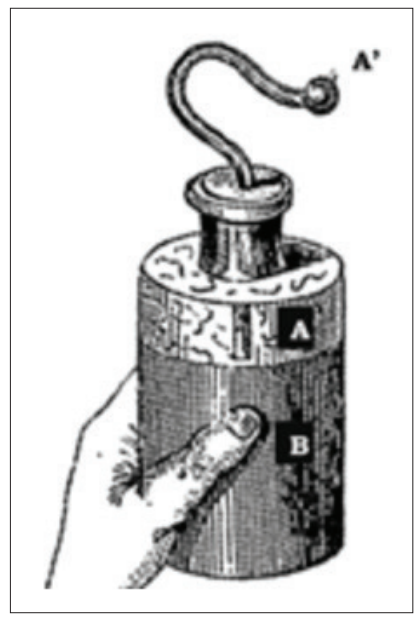

Tanto 1.2- la botella como el aparato electromotor producen conmociones siempre que se encuentre establecido el círculo conductor, o en otras palabras que el circuito esté cerrado. Sin embargo, las contracciones musculares producidas en ambos casos son cortas y momentáneas, estos efectos parecen no ser suficientes para que Volta los asocie una idea de fluido eléctrico continuo. 
Sin embargo, logra asociar la idea de fluido eléctrico tanto al aparato electromotor como a las conmociones producidas por estas descargas acudiendo nuevamente a efectos sensibles. Usando junturas metálicas en su lengua encuentra que en ciertas circunstancias un sabor ácido, o un sabor alcalino, se produce en la punta de la lengua, y permanece, mientras el circuito esté cerrado. Esa sensación es continua, no tiene saltos:

Aplico a la punta de mi lengua una lámina de estaño bien limpia, y más arriba, en el dorso [de la lengua] una moneda de plata u oro; luego, la pongo en contacto con dicha lámina: al instante se excita en la punta de mi lengua una sensación más o menos viva, la de un sabor amargo bien definido, que continúa e incluso aumenta mientras dura la comunicación de ambos metales. En lugar de una hoja sólida de estaño, utilizo más ventajosamente una hoja delgada de este metal, o un pedazo de este papel de plata que he mencionado anteriormente. Aquí hay un fenómeno muy llamativo: pero lo que es más importante, es que al hacer el experimento de manera inversa, es decir aplicando al dorso de la lengua estaño y plata al final [en la punta], se siente aquí un sabor completamente diferente, un sabor ardiente tirando a lo amargo, de una tersura más alcalina que ácida.

No hay duda de que esta sensación diferente viene del hecho de que el fluido eléctrico entra en un extremo y penetra en la punta de la lengua, y en el otro lado sale; yo creo que he determinado cuál de los dos sabores es la entrada y la salida de dicho fluido. Por tanto, el sabor ácido es el que se produce al entrar y el otro alcalino o casi alcalino al salir: así, el estaño hace pasar el fluido eléctrico hacia la parte que éste cubre y, por el contrario, la plata hace salir el mismo fluido hacia los puntos que están en contacto con ella. Para determinar esto mismo he recurrido a la electricidad artificial [máquina electrostática]: apliqué la punta de la lengua al gran conductor de la máquina electrificada a veces de manera positiva, a veces de manera negativa, y sentí claramente los mismos dos sabores, el ácido por la electricidad positiva, el otro sabor acre, casi alcalino, por el negativo. VOLTA $(1792$, p. 127)

Nótese que la electrificación positiva y negativa ya establecida en el campo de la electrostática, ahora es usada por Volta para asociar la electrificación positiva al sabor ácido y la electrificación negativa al sabor alcalino. Esta comparación hace suponer que la electricidad producida por las máquinas electrostáticas podría ser semejante a la electricidad producida por la juntura bimetálica (pila: estaño - plata). Además de compartir conmociones, se comparten también los sabores para cierto tipo de electrificación.

Al preguntarse si los sabores podrían ser producidos por los metales, o por el conductor líquido que actúa en la pila, propone un nuevo experimento: pone un trozo de madera embebido en líquido entre el conductor y su lengua, nuevamente el sabor ácido se produce del lado que él ha llamado positivo, y el sabor alcalino del lado que ha llamado negativo, la sensación durará el tiempo que se mantenga el circuito. Con esto concluye que el sabor es provocado por la distribución de la pareja de metales y no por los metales individuales. 
Que los sabores producidos por el paso del fluido eléctrico sean una sensación continua mientras el circuito esté cerrado permite suponer el movimiento del fluido eléctrico a lo largo del círculo conductor. Además, al diferenciar los sabores y con ello establecer la entrada del fluido en uno de los metales [estaño] y la salida en el otro [plata], Volta atribuye una dirección a tal movimiento: el flujo se dirige de positivo a negativo entre los dos metales, el flujo tiene una direccionalidad definida desde $(+\mathrm{E})$ hacia $(-\mathrm{E})$ asumiendo las convenciones que se habían establecido en el campo de la electrostática.

... para excitar el sabor ácido de la lengua, al mantener la punta aplicada al primer conductor de la Máquina [pila] con electrificación positiva (+ E), y para excitar el sabor alcalino, al mantenerla aplicada al otro conductor unido a las almohadillas [húmedas] y electrificado negativamente ( $\mathrm{E})$, se necesita que la máquina eléctrica ponga en movimiento una corriente de fluido muy abundante. Se requiere una corriente tan abundante, e incluso más, tan continua, y sin salto, para que permanezca sensible al órgano del tacto, para excitar ardores en el ojo, o en las heridas de delicado sentido, etc... $\left(^{*}\right)$.

(*) Estas sensaciones de gusto en la lengua, de ardor en el ojo, etc. son débiles en los primeros momentos, y van creciendo con la continuación de la corriente eléctrica, siempre abundante y del mismo tenor. Por el contrario, el ardor, insensible durante muy pocos segundos, al prolongar la experiencia comienza a hacerse sentir, crece y, finalmente, llega a ser casi insoportable al cabo de uno o dos minutos. VOLTA $\left(1918\right.$, p. 485) ${ }^{9}$

Con estas palabras Volta fortalece la idea de una electrificación positiva y negativa que pone en movimiento la corriente eléctrica, que requiere por lo tanto describir el fluido eléctrico como fluido continuo y en movimiento- que se incrementa al disponer un mayor número de parejas metálicas.

Pancaldi señala que desde 1792 Volta asumió que la electricidad manifestada por las contracciones de la rana derivaba de un desequilibrio del fluido eléctrico causado por las uniones de arcos bimetálicos (PANCALDI, 1990, p. 129) con los que Galvani conectaba sus ranas, éstos son el origen de la electricidad llamada animal. La electricidad se explica en términos de un desbalance entre el poder de los metales que causa el movimiento del fluido, detectable tan pronto como las placas metálicas son conectadas en un círculo a través del animal, la pata de la rana o la lengua de Volta. Si los metales son iguales, o si se quita uno de los arcos metálicos, los efectos no son perceptibles, esto indica que no se origina el fluido eléctrico, y se fortalece la explicación de su desequilibrio, que hoy día reconocemos como la diferencia de potencial eléctrico.

Así pues, través de estas sensaciones y la producción de nuevas y diversas experiencias, Volta deduce que la electricidad que se manifiesta en las botellas de Leyden, en las patas de las ranas y en los distintos circuitos son electricidades de la misma naturaleza, si comparten junturas de pares bimetálicos, los mismos efectos y la posibilidad de incrementar la cantidad de electricidad (producida o almacenada). Dicho en otras palabras, se concluye que en estos fenómenos distintos actúa una sola clase de electricidad. Se resalta en este punto cómo se llega a una unificación de las diversas formas de entender la manifestación de la electricidad. 
En este caso, las conceptualizaciones que ya se habían alcanzado en el campo de la electrostática como la electrificación positiva o negativa, la resistencia o la tensión eléctrica son adaptables a la concepción de electricidad en movimiento que aparece en los fenómenos con los que ahora Volta está tratando (PANCALDI, 1990, p.158). Utilizar tales conceptos se articula con las nuevas concepciones en este proceso de relacionar dos o tres clases de electricidades diferentes. (MALAGON, J.F. 2018b, p. 12)

Estas conexiones derivaron en una concepción unificada de la electricidad, y de este modo, Volta definió que la electricidad es una corriente continua mantenida en movimiento a lo largo de un círculo formado por los diferentes conductores que están distribuidos sin interrupción, es una magnitud aditiva, que como veremos adelante, depende de los conductores usados.

Llegar a una definición, como se puede apreciar, implica delimitar diferentes aspectos de lo observado, establecer proposiciones lógicas inferidas a través de diversas organizaciones, indica estabilizar aquello que se ha conceptualizado para que adquiera una unidad de significado relativamente permanente. Por lo tanto, para nosotros, las definiciones o los conceptos no han de ser los puntos de partida para la enseñanza, son lugares a los que hay que llegar, y estos procesos no corresponden a un nivel específico del aprendizaje, o a un grupo etario particular, son procesos que se dan en todos los niveles del aprendizaje, incluso en todas las áreas del conocimiento. De ahí que, nos parezca tan necesario y pertinente el estudio de estos aspectos. ¿Por qué esto sería importante para la enseñanza?

Así pues, este concepto de electricidad como fluido en movimiento es una gran síntesis o unificación en el campo de la electricidad que permite comprender fenómenos en apariencia distintos a partir de unos efectos compartidos y de unas características semejantes. Esta semejanza concreta magnitudes con las cuales es posible describir, explicar y cuantificar efectos, una de estas magnitudes es la tensión eléctrica, que describiremos en el siguiente apartado.

\subsection{La tensión eléctrica de las pilas}

Detallemos cómo es el proceso de concretar la magnitud de la tensión en el caso de las pilas, y cómo la producción de efectos posibilita la construcción de generalizaciones. Al respecto OSTWALD (1912) presenta una descripción del trabajo de Volta:

si se construye una pila A F B F C y una pila A F C (F representa el conductor húmedo), ellas tendrán ambas la misma tensión. Al final, Volta observa como ya hemos dicho, que se puede asociar entre sí tantos metales como se quiera, de todas las maneras posibles, sin obtener jamás la mínima variación de tensión. ${ }^{10}$ (OSTWALD, 1912, p. 66)

Esta primera ley empírica: No importará el número de placas intermediarias siempre que haya la distribución metal - conductor húmedo - metal y los metales extremos de la pila sean A y $C$, la tensión seguirá siendo la que producen los metales $A$ y $C$, no es una ley arbitraria.

en la pila A F B F C, el metal ubicado en el centro está en contacto por los dos lados con el mismo conductor húmedo, y da origen, por consiguiente, a dos tensiones iguales; pero estas tensiones, al estar en sentidos contrarios, se neutralizan. Quedan 
por considerar las superficies de contacto A F y F C. Las tensiones de estas superficies de contacto están igualmente en sentidos contrarios; la tensión resultante es entonces igual a la diferencia de las tensiones provenientes del contacto de los metales A y C con el líquido. Generalmente, si se intercala en la pila un número cualquiera de metales, al ubicarse cada uno de ellos entre dos conductores húmedos, sus tensiones se anularán recíprocamente, como las de B; la tensión de la pila no dependerá más que de los metales extremos A y C. (OSTWALD, 1912, p. 67)

Volta también aquí recurre a los instrumentos electrostáticos que le permiten comparar las tensiones producidas por distintas distribuciones de pares de metales y conductores húmedos, explica la tensión que se produce en el ensamblaje A F B, donde no hay más contacto que entre metales $[\mathrm{A}$ y B] y el líquido $[\mathrm{F}]$ :

[Volta] hacía resaltar que es obligatorio emplear un conductor metálico para unir este ensamblaje al electrómetro por medio del cual se mide la tensión. Si este conductor está hecho de metal A, se obtiene una pila A F B A; por consiguiente, el electrómetro debe indicar la tensión B A. (OSTWALD, 1912, p. 67)

En este punto, las tensiones entre metales al ser conectados uno tras otro podrían ser sumadas. Volta presenta una manera de organizar los efectos que inicialmente describía según la intensidad de su sensación.

Observó además de esto: $\mathrm{A}, \mathrm{B}$ y $\mathrm{C}$ son tres metales, (...) si se combinan de dos en dos con un conductor húmedo, ellos dan como resultado las tensiones $(A, B),(B, C)$, $(\mathrm{A}, \mathrm{C})$; y se tendrá siempre la relación: $(\mathrm{A}, \mathrm{B})+(\mathrm{B}, \mathrm{C})=(\mathrm{A}, \mathrm{C})$; es decir, si se agrega a la tensión de $\mathrm{A}$ y $\mathrm{B}$, asociada con el conductor húmedo, la de $\mathrm{B}$ y $\mathrm{C}$ asociada a este conductor, la suma obtenida es igual a la tensión de A y $\mathrm{C}$ asociada con este mismo conductor. (OSTWALD, 1912, p. 65)

Esta forma de medir la intensidad de los efectos da cuenta de otro momento de síntesis teórico. Al establecer la magnitud de la tensión es posible definir las condiciones para la producción de efectos de diferente intensidad, que no dependen de la sensibilidad de quién sufre los espasmos musculares.

Si bien la intensidad de los efectos podría ordenarse desde los más débiles a los más fuertes en relación con las sensaciones, en este momento de la construcción del fenómeno esa organización perceptual se vincula al uso de ciertos instrumentos, representaciones y símbolos que llevan a establecer generalizaciones. Operaciones como: $(\mathrm{A}, \mathrm{B})+(\mathrm{B}, \mathrm{C})=(\mathrm{A}, \mathrm{C})$, se establecen en el terreno de la representación simbólica que sintetiza el resultado experimental y representa la operación lógica que lleva a la generalización. En este caso, la tensión no sólo está ligada a la intensidad de las sensaciones, sino que se encuentra en un nivel de abstracción que ahora depende de esta relación operacional, esto es: en la unión de diferentes pares de placas hay una adición de estas intensidades según su distribución. En la actualidad, reconocemos este nivel de operación en la organización de los circuitos cuando se encuentran en serie o en paralelo. 
Una pila conformada por pares de metales y conductores húmedos: $A B$ $\mathrm{F}$ AB F AB, donde $\mathrm{AB}$ son dos metales y donde $\mathrm{F}$ es el conductor húmedo, podría tener intensidades mayores de tensión de acuerdo con el número de pares metálicos que la conforman. Pero si la pila se configura de la forma AB F BA la tensión producida por los pares de metales se anula, en tanto las acciones que se desarrollan al interior $\mathrm{AB}$ y $\mathrm{BA}$ son iguales y opuestas, además en BFB y en los extremos AA al ser metales iguales no hay acción.

Por estas organizaciones nosotros consideramos que el establecimiento de la magnitud es una síntesis teórica, ésta generaliza, no en el sentido estricto de una ley o un principio, sin embargo, guarda o abriga leyes empíricas:

En las pilas distribuidas A F B

La tensión obtenida por una pila se establece entre los metales de diferentes extremos de la pila.

Las tensiones que generan los pares de metales intermediarios cuando se apilan en serie se suman.

Las tensiones se anulan cuando se juntas pares de metales iguales, o cuando las acciones de pares de metales diferentes son iguales y opuestas.

Al elaborar esta síntesis que está intrínsecamente ligada al proceso de experimentación se amplía la mirada que se tenía sobre la electricidad. Se puede afirmar, entonces, que los procesos de experimentación desarrollados por Volta son, también, procesos de formalización que se adquieren para un cierto campo fenomenológico.

\section{PROCESOS DE TEORIZACIÓN EN CIENCIAS}

Hasta aquí se ha mostrado que los procesos de teorización implican un entrelazamiento dinámico entre las percepciones, los modos de hablar asociados a esas percepciones, y las formas de actuar con respecto a ellas. Ese entrelazamiento visible en el trabajo de Volta atribuye una similitud entre los efectos producidos por la botella de Leyden, por la torre de pares de metales y por los tejidos animales; la duración de las conmociones y las condiciones para que éstas se produzcan permiten expresar que el fluido eléctrico es de la misma clase. Y es así como el fluido eléctrico resulta de una asignación lingüística para dar cuenta de condiciones de producción de ciertos efectos, y viceversa.

Siguiendo a DUHEM (2003, p. 25), este ejercicio de síntesis implica un proceso de abstracción que destaca lo común de un conjunto de hechos concretos, aislados y diferentes entre sí, para ser presentado en una proposición única que se hará general. Así, los procesos de síntesis teórica son elaboraciones intelectuales complejas que implican el reconocimiento de hechos concretos y de sus condiciones de producción, para proceder a organizar efectos, comparar, clasificar, etc. En este proceso la producción de símbolos y términos es la forma de explicitar esta organización.

Asimismo, la percepción vinculada con actos de habla ${ }^{11}$ se concreta, pero no de cualquier manera, se relaciona con actos de pensamiento, con un cierto contenido conceptual de lo ya conocido. ${ }^{12}$ La percepción es una operación 
intelectual ligada al pensamiento sobre el mundo, no es pasiva, ella misma es el motor de la producción de efectos sensibles.

Observamos que incluso una percepción es una síntesis teórica cuya fragmentación en dato sensible resulta ser un ejercicio que llevaría a una infinitud de descomposiciones que resultaría imposible describir, es imposible descomponer una percepción y convertirla en un conjunto de sensaciones. En estos procesos, la percepción no es colocada como causa o consecuencia, ni principio de los procesos ni producto de esta. De ahí que es necesario que significados y símbolos, la forma y la materia de la percepción, se relacionen desde el comienzo:

Cuando examinamos lo que nosotros deberíamos decir cuando pensamos qué palabra debemos usar en qué situaciones, estamos mirando de nuevo no solo las palabras (o 'significados', cualesquiera que sean) sino también las realidades en las que usamos las palabras para hablar: estamos usando una conciencia aguda de las palabras para agudizar nuestra percepción de los fenómenos, aunque no como árbitro final de éstos (AUSTIN, 1970, p. 182).

En consecuencia, establecer campos semánticos desde los cuales abordar las disciplinas científicas es un proceso que ha de estar ligado a cómo se manifiesta la percepción. En la naturaleza, dice Duhem, solo hay hechos, la ley única que los reúne no es más que la copia de caracteres comunes entre todos estos hechos, o mejor [la copia] de caracteres comunes que nos interesan, y que están resumidos por nuestra abstracción, y solo ella [la ley], entrará en la ciencia en lugar de múltiples hechos (DUHEM, 1992, p. 445). Son estos resúmenes los grandes avances de los que se constituye la ciencia, en particular, y el conocimiento en general. Implica que las teorías guardan una relación con la experiencia, aunque no son la experiencia: las experiencias con el mundo se interpretan, se seleccionan, se tematizan, se relacionan con otras experiencias, y en estos procesos se adquieren sentidos y significados, tomamos conciencia del objeto de estudio en el mundo y el objeto es objeto para nuestra conciencia, por cuanto se ha constituido a la luz de esquemas conceptuales que ya tenemos en el pensamiento y que hacen parte de nuestro saber.

Desde nuestra perspectiva fenomenológica, no limitamos la percepción a las primeras impresiones en nuestros órganos de la sensación, no entendemos la percepción como una impresión cerebral - orgánica; la entendemos como el progreso en la organización, tematización y comprensión de los fenómenos que genera nuevas experiencias, las cuales vienen acompañadas de nuevas formas de percepción que nos llevan a consolidar nuevos arreglos experimentales, a establecer diferentes relaciones formales y a la producción de nuevas afirmaciones que enriquecen los puntos de vista, amplían las percepciones, generan argumentaciones, en un proceso creativo mediante el cual el conocimiento no se agota y se extiende. En consecuencia, no son los otros, en este caso los científicos, quienes nos dicen cómo debemos ver el mundo, somos nosotros que en diálogo con ellos aprehendemos de esas cosmovisiones que se estabilizan en la cultura.

Por estas razones, hemos tratado la experimentación como mucho más que la observación de un fenómeno, consideramos que acompaña la interpretación teórica del fenómeno de estudio, del mismo modo que la interpretación teórica 
deriva en nueva experimentación. En las descripciones que Volta nos presenta mediante sus experimentos, los conceptos como fluido eléctrico o tensión adquieren significación en tanto la disposición del montaje permite establecer las condiciones para producir circuitos eléctricos. La distinción de efectos sensibles y la organización de las condiciones para producirlos son etapas dentro del proceso de teorización y de consolidación de conceptos y leyes establecidas sobre las condiciones de los circuitos.

En otras palabras, la teorización que constituye la unidad de los objetos percibidos y que da significado a los datos perceptuales es una síntesis intelectual. Por eso, al abordar un fenómeno para su estudio, hacemos énfasis en la descripción detallada y en la explicitación y profundización en la interpretación.

Sin embargo, sintetizar las elaboraciones y producir generalizaciones son operaciones que tienen que ver con la relación entre distintos campos fenomenológicos: electrostática, electricidad animal y electricidad dinámica. La cualidad estudiada y las generalizaciones que se adquieren tienen un rango de aplicación no sólo para el campo fenomenológico referido sino extrapolable a otros campos. En el caso que hemos expuesto anteriormente, el estudio de la producción de efectos por la juntura de metales implicó tanto la organización de los fenómenos eléctricos nuevos (con la definición de la electricidad, la concepción de circuito, corriente y tensión) y estas organizaciones se extrapolaron y vincularon con los fenómenos electrostáticos. Así chispas, conmociones, ardores, la electricidad animal, todas fueron agrupadas como efectos de la acción continua de la corriente eléctrica.

\section{CRITERIOS E IMPLICACIONES PARA LA ENSEÑANZA}

Recapitulando, en relación con el trabajo de Volta, resaltamos el vínculo indisoluble entre: la percepción, la producción de efectos sensibles, el lenguaje que los describe, la producción y reproducción de experiencias, y los momentos de generalización que ocurrieron en cada fase específica del estudio de la pila. Estos aspectos ejemplifican una forma de proceder y de razonar en el campo de las ciencias claramente fenomenológica: sobre aquello que se manifiesta como fenómeno hay una conciencia que actúa para hacer inteligible y reproducible cierto atributo del mundo, la conciencia en las acciones sobre el fenómeno permite la producción de conocimiento.

Para nosotros tal dinámica en la producción de conocimiento nos ha llevado a considerar, entonces, tres criterios fundamentales para guiar los procesos de enseñanza, entendiendo como criterios aquellos aspectos que permiten la toma de decisiones para proceder, en nuestro caso, en las propuestas para la enseñanza de las ciencias.

\subsection{El primer criterio consiste en asumir la acción de tematizar.}

Consideramos que los docentes somos responsables de aquello que se selecciona como objeto de enseñanza: aquello que se enseña ha de ser identificado y establecido como un problema de estudio, primero para el docente, y posteriormente, para sus estudiantes quienes se apropian del problema y lo reformulan. 
Tematizar consiste entonces en problematizar, en hacerse preguntas, en razonar sobre aquello que se manifiesta como fenómeno. Hemos presentado el caso del efecto Volta como un ejercicio de tematización donde las preguntas, los razonamientos, las organizaciones que se establecen hacen parte del cómo tematizar.

Así, nos parece necesario acudir a la historia de la ciencia y seleccionar de ésta episodios históricos que permitan establecer casos de estudio para comprender las formas de organización, entender las preguntas y contextos donde éstas se producen. Estas son acciones orientadas desde las preguntas de orden disciplinar y pedagógico que el docente se puede plantear en su necesidad de comprender eventos del mundo.

Tematizar, entonces, es la acción que permite que el docente ya no reemplace el estudio sobre los fenómenos por los productos o resultados de la ciencia, como señalábamos al principio de este artículo, porque ahora, al plantearse sus propios problemas, el docente puede entrar en diálogo con la ciencia, puede asumir qué es lo que ha de ser enseñado, $y$, además, tiene una finalidad para su enseñanza.

\subsection{El segundo criterio consiste en asumir la acción de experimentar.}

Así, desde esta perspectiva, la actividad experimental se constituye en eje central para desarrollar procesos de enseñanza y de aprendizaje de las ciencias, por cuanto esta actividad permite producir efectos e identificar cualidades que son susceptibles de ser ordenadas, clasificadas, analizadas y/o cuantificadas mediante instrumentos que se ingenian para ello, y sobre su análisis los sujetos amplían su experiencia, lo cual propicia la comprensión sobre los fenómenos estudiados en las clases.

Consideramos que, tanto el diseño experimental que interviene sobre las magnitudes de los efectos, como el estudio de la instrumentación que las sintetiza y cuantifica, permiten conceptualizar en diferentes momentos de la actividad, es decir, permiten la producción de afirmaciones cada vez más argumentadas sobre lo que se observa, se establecen interpretaciones y descripciones estructurantes para referirse a los hechos que se manifiestan con la experimentación. De este modo, a partir de unas acciones sobre lo material, se opera cognitivamente bajo formas lógicas, se despliegan las organizaciones teóricas con las cuales describimos y comprendemos el mundo. Esta idea, que es como lo señala GARCÍA (1997) la tesis más original de Jean Piaget en su epistemología constructivista queda expresada en el siguiente párrafo:

Para una epistemología constructivista no hay conocimiento de la realidad empírica que se va desarrollando como lo quieren explicar los aprioristas o los empiristas, y al cual se le aplica luego una lógica, y una matemática, generadas de manera autónoma. La construcción es simultánea. La lógica comienza con relaciones e inferencias elementales que se establecen ya en el nivel sensoriomotor, y que se prolongan en las acciones más generales que se pueden ejercer sobre los objetos: juntarlos, agruparlos y ordenarlos. Sin acciones sobre los objetos no hay lógica, pero la lógica no se genera a partir de los objetos. (GARCÍA, 1997, p. 47)

Resaltamos esta simultaneidad entre las acciones y la organización lógica sobre ellas; es la tesis central sobre la cual se constituye también esta perspectiva 
fenomenológica que sugiere, como bien lo expresa RAMÍREZ (2013), un entrelazo donde "la percepción es el acontecimiento primordial porque es la forma primigenia y permanente del encuentro del sujeto con el mundo” (RAMÍREZ, 2013, p. 53).

Esto implica considerar la experimentación como una actividad inmersa en el proceso de formalización de las comprensiones que se adquieren en torno a un campo fenomenológico, en ese entrelazo perceptivo sujeto-mundo, que otorga al conocimiento un lugar ubicuo en el ser y en el acontecer. La experimentación no está en oposición o en dualidad con la teorización, no está antes o después, no hay una relación dicotómica teoría-práctica, por el contrario, la teorización es propia de la organización de la experiencia, es la comprensión como aprehensión del mundo.

Desde el punto de vista fenomenológico (RAMÍREZ, 2013, p. 145), y también desde la epistemología genética (GARCÍA, 1997, p. 50), los procesos para el desarrollo del conocimiento tienen como elemento fundamental la construcción de significados. Consideramos, entonces, que una perspectiva fenomenológica posibilita que el estudiante de ciencias, sin importar su nivel académico, piense y actúe sobre aquello que se manifiesta como problema o fenómeno de estudio, y que, si los docentes de ciencias tienen formación en esta dirección, la ciencia que se estudia en los espacios escolares adquiere un mayor campo de significados.

Esto nos lleva a identificar que, en términos cognitivos, la experimentación orienta procesos complejos de razonamiento inductivo que llevarán a generalizaciones establecidas sobre lo perceptible, como aquellos procesos que le permitieron a Volta hacer descripciones cada vez más detalladas sobre los efectos de su pila. Pero, como señala GARCÍA (1997):

[...] esto no basta para fundamentar el conocimiento «la cuestión de la generalidad no puede presentarse, desde el punto de vista constructivista, en términos de la presencia o ausencia de un cierto predicado: lo que es más o menos general es todo el sistema de relaciones». Por eso introduce Piaget el concepto de generalización constructiva. La generalización constructiva consiste en establecer nuevas formas de relación que conducirán a nuevas estructuras, lo cual difiere de la asimilación de nuevos contenidos (empíricos) a formas y estructuras ya constituidas (GARCÍA, 1997, p.52)

Es éste el proceso de razonamiento inductivo que observamos en la conformación del fenómeno voltaico, una generalización dada por el establecimiento de nuevas formas de relación que permitieron a Volta transitar desde las experiencias con la "electricidad animal", examinar casos distintos como las experiencias con la "electricidad estática”, y obtener "electricidad dinámica” en los apilamientos de metales o conexiones semejantes a aquellas que ya se hacían en otros estudios de la electricidad; finalmente, surgieron nuevos problemas de estudio que se concretaron en: pilas de diferentes clases, corrientes eléctricas más intensas, aparatos más sensibles, efectos nuevos de descomposición de sustancias, etc. De este modo, lo común de estos múltiples casos fue la acción de una forma de electricidad continua, que estableció el vínculo para la explicación de fenómenos aparentemente distintos en términos de los mismos conceptos: tensión, cantidad de electricidad, conductividad, fuerza electromotriz, etc. 
Por esto, consideramos relevante el papel del razonamiento inductivo en la construcción de conocimiento y, por lo tanto, en la enseñanza de las ciencias. Esta forma de razonamiento, como vemos, permite constituir síntesis (resúmenes de la experiencia) que nosotros consideramos de carácter teórico, ${ }^{13}$ porque son proposiciones lógicamente establecidas.

\subsection{El tercer criterio consiste en reconocer los procesos de teorización que se derivan de las acciones anteriores.}

La perspectiva fenomenológica, implicada en el anterior criterio, da preponderancia a la actividad de organización de los efectos sensibles y resalta los procesos de teorización que pueden derivarse de las acciones intencionadas desarrolladas en la actividad experimental. Reconocer procesos de teorización en la enseñanza significa que se relieva la necesidad de considerar que las acciones en el aula tienen diferentes grados de abstracción; la generalización de efectos, el establecimiento de relaciones entre efectos y conceptos, ubicados en otras organizaciones teóricas, hacen parte del proceso de organización que los sujetos despliegan para la comprensión de los fenómenos. Esta mirada se desliga de las propuestas de enseñanza que consideran a los sujetos como pasivos frente a un conocimiento ya establecido sobre el mundo.

Este criterio implica que en el proceso de enseñanza el profesor privilegie acciones donde los estudiantes:

- Expliciten las razones que los llevan a identificar aspectos comunes de las condiciones y de los efectos producidos en la actividad experimental desarrollada. - Identifiquen y comuniquen generalizaciones a partir de la actividad intencionada que orienta las acciones que realizan sobre los objetos.

Esto obliga que el profesor conciba que los estudiantes podrían tener diferentes argumentos y estrategias para obtener esas generalizaciones, para representarlas y discutirlas a la luz de las otras experiencias. Por tanto, también será necesario que:

- Reconozca que los procesos de teorización en el aula no son inmediatos: Se produce un nivel de organización que permite pasar de la experiencia sensible a la producción de generalizaciones [magnitudes, relaciones entre magnitudes, unificación de fenómenos, establecimiento de analogías, etc.].

- Contraste los esquemas conceptuales que involucran sus estudiantes en la actividad de organización con otras organizaciones teóricas que sustentan experiencias anteriormente abordadas o que requieren ser tratadas en el aula.

Estos criterios han estado presentes en el diseño e implementación de talleres y cursos dirigidos a profesores en formación inicial, posgradual y en servicio. Las actividades que se realizan han resultado formativas para quienes tienen algunos referentes sobre el efecto voltaico y las magnitudes abordadas, así como para quienes por primera vez se enfrentan a este tipo de fenómeno, pues la propuesta posibilita que los participantes elaboren experiencias, relaciones lógicas y significados propios ligados con los efectos producidos por ellos mismos.

Las anteriores consideraciones han respaldado otras propuestas de trabajo que hemos desarrollado donde se logran clasificaciones, ordenaciones, mediciones 
de diferentes intensidades de una cualidad (AYALA MANRIQUE y otros, 2011); sin embargo, es necesario también enriquecer estas propuestas para lograr generar procesos de teorización. En el momento sabemos que a través del estudio de una cualidad (como las contracciones ocurridas por el fluido eléctrico, lo frío, lo ácido, lo básico, etc.) sobre la cual se pueden operar clasificaciones u ordenaciones, o se pueden construir formas y aparatos de medida, también se puede organizar una clase de fenómenos en relación con sus efectos producidos, que en la mayor parte de los casos pertenecen a un campo fenomenológico distinto (electricidad animal, voltaica y estática; dilatación de sustancias; cambio de color; movimiento etc.).

En consecuencia, cuando nos proponemos desplegar actividades para el aula de clase, hacemos énfasis en la construcción y comprensión de fenomenologías. La producción de efectos, y con ello, el proceso de organización que los sujetos pueden hacer cuando desarrollan sus propios análisis son los aspectos para privilegiar con toda la dinámica conceptual que tiene lugar en los escenarios de la enseñanza-aprendizaje de las ciencias.

\section{AGRADECIMIENTOS}

Este artículo se produce en el marco del proyecto de investigación DFI 430 16 Procesos de Teorización: Síntesis y Campos Fenomenológicos; y del proyecto de investigación DQU-478-18 Relaciones de Equivalencia y Construcción del Campo Fenomenológico: El caso de la electroquímica.Ambos proyectos internos de la División de Gestión de Proyectos - Centro de Investigaciones, Universidad Pedagógica Nacional de Colombia.

\section{REFERENCIAS}

AUSTIN, J. L.Philosophical Papers: How to Talk. Oxford: Oxford University Press, 1970.

AYALA, M.M., MALAGON, J.F., SANDOVAL, S. Ellenguaje y la construcción de fenomenologías: el caso del efecto Volta. Revista Brasileira da historia da ciencias.7(2), 203 - 213, 2014.

BRUNER, J. Actos de Significado, (Trads: Gómez Crespo y Linaza) Madrid: Alianza Editorial. 1991

CAMACHO GONZÁLEZ J., IZQUIERDO M., SOLSONA N. The History of Science in Teacher Training Programs: A Series of Contributions and Debates for the Teaching of Electrochemistry. In: Prestes M., Silva C. (eds) Teaching Science with Context. Science: Philosophy, History and Education. Springer, p. 253, 2018.

DUHEM, P. L’évolution de la mécanique. Paris: Librairie Philosophique J. Vrin. 1992.

DUHEM, P. La teoría física su objeto y su estructura. Barcelona: Herder editorial, 2003.

ERDURAN; DAGHER. Reconceptualizing the Nature of Science for Science Education. Netherlands: Springer, 2014.

GALVANI, L. De viribus electricitatis in motu musculari. (E. Benassi, Trad.) Bolonia. 1937. Recuperado en 2019, de https://web.archive.org/web/20070822092312fw_/http:/ / cis.alma.unibo. it/galvani/textus.html. 
Marina Garzón Barrios | Liliana Tarazona Vargas | Sandra Sandoval Osorio José Francisco Malagón Sánchez | María Mercedes Ayala Manrique

GANOT A.Cours de physique purement expérimentale. París: Lacroix et Baudry, 1859.

GARCÍA, R. La epistemología genética y la ciencia contemporánea. Barcelona: Gedisa, 1997.

HEILBRON, J. A propos de l'invention de la bouteille de Leyde. Revue d'histoire des sciences et de leurs applications, 19(2), 133-142, 1966.

M.R. MATTHEWS (ed.), International Handbook of Research in History, Philosophy and Science Teaching, (C) Springer Science+Business Media Dordrecht p.343, 2014.

MALAGÓN, J.F., AYALA, M.M., SANDOVAL, S. El experimento en el aula. Comprensión de fenomenologías y construcción de magnitudes. Bogotá: Universidad Pedagógica Nacional, 2011.

MALAGON, J.F., AYALA, M.M., SANDOVAL, S. Construcción de fenomenologías y procesos de formalización. Un sentido para la enseñanza de las ciencias. Bogotá: Fondo Editorial Universidad Pedagógica Nacional, 2013.

MALAGON, J.F., AYALA, M.M., BARRIOS, M., SANDOVAL, S. Constitución de un dominio fenomenológico para la enseñanza de las ciencias. Implicaciones desde el estudio del caso del fenómeno voltaico. Ensaio. Pesquisa em educação em ciencias, 20, 1 - 23, 2018 b.

OSTWALD, F. W. L'evolution de l'électrochimie. París: Librairie Félix Alcan, 1912.

PANCALDI, G. Electricity and life. Volta's path to the battery. Historical Studies in the Physical and Biological Sciences. 21(1), p. 123-160, 1990.

RAMÍREZ, M. La filosofía del quiasmo. Introducción al pensamiento de Meleau-Ponty. México: Fondo de Cultura Económica, 2013.

SANDOVAL, S., MALAGON, J. F., GARZON, M., AYALA, M.M., TARAZONA, L. Una perspectiva fenomenológica para la enseñanza de las ciencias. Bogotá: Universidad Pedagógica Nacional, 2018a.

VOLTA, A. "Sobre la electricidad excitada por el simple contacto de substancias conductoras de distintas especies (Carta)”. En: Sallent Del Colombo, E. (2000). Llull: Revista de la Sociedad Española de Historia de las Ciencias y de las Técnicas. 23 (48), p. 763-784, 1800a.URL=https:// Dialnet.unirioja.es/descarga/articulo/2961105.pdf.

VOLTA, A. On the electricity excited by the mere contact of conducting Substances of different kinds. Philosophical Transactions of the Royal Society of London. January 1, 90, p. 403-431. 1800b.

VOLTA, A. Le Opere di Alessandro Volta (Vol. I). Milano: Ulrico Hoepli_ Edittore-Libraio della Real Casa. 1918.

\section{NOTAS}

1 Es poco habitual que en las clases de ciencias se estudien los presupuestos teóricos, las discusiones y los experimentos que sustentan la estructura atómica de la materia, siendo un capítulo tan importante en la historia de las ciencias. Por el contrario, se asume que la estructura atómica es algo establecido que se comprende cuando se dibujan o representan los modelos atómicos. Menos aún se estudian los problemas y experimentos que dan origen a la idea de ion. Para profundizar en esta afirmación se sugiere ver los trabajos de CAMACHO GONZÁLEZ J., IZQUIERDO 
M., SOLSONA N. (2018) The History of Science in Teacher Training Programs: A Series of Contributions and Debates for the Teaching of Electrochemistry. M.R. MATTHEWS (2014), International Handbook of Research in History.

2 Pese a que en el campo de la ciencia estas mismas imágenes electrónicas se hayan organizado sobre la base de importantes experimentos y conceptualizaciones.

3 Entendemos la percepción dentro de un complejo mental y lingüístico. La percepción no es el simple dato-sensorial, la percepción se ubica dentro de un entrelazo sujeto-mundo donde ya se han organizado actos de significado. Este en un aspecto que se precisará más adelante.

4 La controversia Volta - Galvani es una de las controversias más citadas en el campo de la electricidad. Para profundizar en este aspecto ver el libro de MARCELLO PERA (1992): The ambiguous frog: The Galvani-Volta Controversy on animal electricity. Princeton University Press.

5 Hemos resaltado en negrilla aquellas palabras o expresiones que destacamos como importantes dentro del análisis.

6 El término de intensidad se refiere en este caso a la intensidad de la sensación generada por el fluido eléctrico; en el trabajo de Volta no hay aún un uso diferenciado de las magnitudes tensión y corriente eléctrica, magnitudes que hoy en día también podrían ser asociadas a esta intensidad de la sensación. Volta inicialmente se refiere a la intensidad de los efectos que sugiere van variando en cantidad si la disposición de metales va aumentando o disminuyendo con respecto a condiciones de número de pares de metales y continuidad de la disposición. PANCALDI, (1990, p. 130-133)

7 Las botellas de Leyden habían sido construidas durante 1746 para almacenar electricidad. Son recipientes de vidrio (Figura 3) que tienen en su interior laminillas metálicas A (oro, estaño, etc.) y en el exterior un recubrimiento metálico B (aluminio o estaño). Desde la parte superior de las botellas entra un gancho metálico con una esfera metálica que sirve de contacto A'. Estas botellas fueron comúnmente utilizadas para almacenar la electricidad producida por diversas máquinas electrostáticas y se descargan en forma de chispas suficientemente intensas como para matar pequeños ratones. Se popularizaron en las ferias. Para conocer más sobre su origen, ver: A propos de l'invention de la bouteille de Leyde de John L. HEILBRON (1966, p.133-142).

8 Traducción del texto en francés. Lettera prima a Marino Van Marum. Agosto 1792. VOLTA (1918)

9 Traducción del texto en italiano Mémoire sur l'electricité excitée par le contact. VOLTA (1918)

10 Como se dijo anteriormente, en el trabajo de Volta no hay una distinción entre tensión y corriente eléctrica como magnitudes independientes (PANCALDI, 1990, p. 130-133). Volta describe el poder de los metales para empujar el fluido eléctrico. Hoy día tal poder lo nombramos como Tensión eléctrica o Voltaje. Mientras que a la intensidad del fluido es la magnitud que asociamos con la intensidad de corriente, de ahí que uno de nuestros objetivos de enseñanza sea organizar estas magnitudes para diferenciarlas, como se presentará más adelante (ver p. 23 de este documento).

11 De acuerdo con la filosofía lingüística de John Langshaw AUSTIN, ver Philosophical Papers: How to Talk. (AUSTIN, 1970, p.134-153)

12 En el sentido que Jerome Bruner señala en su libro Actos de Significado. (BRUNER, 1991, p. 33-34)

13 En los procesos de síntesis hemos identificado algunas relaciones entre campos fenomenológicos que son relaciones de reducción, de unificación y de equivalencia. Ver: AYALA MANRIQUE Y OTROS $2018^{\mathrm{a}}$, 2018b. 
Marina Garzón Barrios | Liliana Tarazona Vargas | Sandra Sandoval Osorio | José Francisco Malagón Sánchez | María Mercedes Ayala Manrique

Submetido em 02/08/2019

Aprovado em 20/02/2020

\section{Contato:}

Universidad Pedagógica Nacional.

Departamento de Física.

Calle 72 Número 11- 86. Edificio B. Segundo Piso.

Código Postal 110221 - Bogotá - D.C. Colombia 